\title{
Trajectory and physical properties of near-Earth asteroid 2009 BD
}

\author{
D. Farnocchia ${ }^{1}$, M. Mommert ${ }^{2}$, J. L. Hora ${ }^{3}$, S. R. Chesley ${ }^{1}$, \\ D. Vokrouhlický ${ }^{4}$, D. E. Trilling ${ }^{2}$, M. Mueller ${ }^{5}$, A. W. Harris ${ }^{6}$, \\ H. A. Smith ${ }^{3}$, and G. G. Fazio \\ ${ }^{1}$ Jet Propulsion Laboratory, California Institute of Technology \\ Pasadena, CA 91109, USA \\ email: Davide.Farnocchia@jpl.nasa.gov \\ ${ }^{2}$ Department of Physics and Astronomy, Northern Arizona University \\ Flagstaff, AZ 86011, USA \\ ${ }^{3}$ Harvard-Smithsonian Center for Astrophysics \\ Cambridge, MA 02138, USA \\ ${ }^{4}$ Institute of Astronomy, Charles University \\ CZ-18000, Prague 8, Czech Republic \\ ${ }^{5}$ SRON Netherlands Institute for Space Research \\ 9700-AV Groningen, The Netherlands \\ ${ }^{6}$ DLR Institute of Planetary Research \\ D-12489 Berlin, Germany
}

\begin{abstract}
We analyze the trajectory of near-Earth asteroid $2009 \mathrm{BD}$, which is a candidate target of the NASA Asteroid Redirect Mission. The small size of 2009 BD and its Earth-like orbit pose challenges to understanding the dynamical properties of 2009 BD. In particular, nongravitational perturbations, such as solar radiation pressure and the Yarkovsky effect, are essential to match observational data and provide reliable predictions. By using Spitzer Space Telescope IRAC observations and our model for the thermophysical properties and the nongravitational forces acting on 2009 BD we obtain probabilistic derivations of the physical properties of this object. We find two physically possible solutions. The first solution shows $2009 \mathrm{BD}$ as a $2.9 \pm 0.3 \mathrm{~m}$ diameter rocky body with an extremely high albedo that is covered with regolith-like material, causing it to exhibit a low thermal inertia. The second solution suggests $2009 \mathrm{BD}$ to be a $4 \pm$ $1 \mathrm{~m}$ diameter asteroid with albedo $0.45 \pm 0.35$ that consists of a collection of individual bare rock slabs. We are unable to rule out either solution based on physical reasoning. 2009 BD is the smallest asteroid for which physical properties have been constrained, providing unique information on the physical properties of objects in the size range smaller than $10 \mathrm{~m}$.
\end{abstract}

Keywords. Radiation mechanisms: general, celestial mechanics, methods: analytical, techniques: image processing, astrometry, ephemerides, minor planets, asteroids, infrared: solar system

\section{Introduction}

Near-Earth asteroid 2009 BD was discovered on 2009 January 16, at a geocentric distance of 10 million kilometers (Buzzi et al. 2009), and it is on an Earth-like orbit.

The orbital geometry makes 2009 BD one of the asteroids with the lowest relative velocity with respect to Earth and it is thus considered a potential target for the Asteroid Redirect Mission (ARM, Mazanek et al. 2103). The current design of ARM requires that the target asteroid has a diameter between $7 \mathrm{~m}$ and $10 \mathrm{~m}$, and a mass of about $500 \mathrm{t}$. 
Little is known about the physical properties of 2009 BD. The absolute magnitude $H=28.43 \pm 0.12$ (Micheli et al. 2012) suggests that 2009 BD is a small object, with a diameter around $10 \mathrm{~m}$ or less. However, the lack of albedo information prevents a more accurate estimate. Tholen et al. (2013) found that 2009 BD has a rotation period somewhat larger than 3 hours.

Due to its small size, indirect information on 2009 BD physical properties comes from the action of nongravitational perturbations, namely the Yarkovsky effect (Vokrouhlický et al. 2000) and solar radiation pressure (Vokrouhlický \& Milani 2000). In this paper we combine the constraints coming from nongravitational perturbations and observations from the Spitzer Space Telescope (Werner et al. 2004) to estimate the physical properties of 2009 BD. For more details see Mommert et al. (2014).

\section{The orbit of 2009 BD}

The osculating orbit of 2009 BD at epoch 2010 January 4 is very close to that of the Earth. The semimajor axis is $1.01 \mathrm{au}$, the eccentricity $4 \%$, and the inclination $0.4^{\circ}$. Because of its orbital configuration 2009 BD stayed in the Earth's neighborhood from 2009 to 2011 thus allowing astronomers to collect astrometric observations. Then, the Earth encounter of June 2011 changed the semimajor axis to 1.06 au and 2009 BD started drifting away from Earth to get closer again only around 2022.

Though the observed arc is quite short, nongravitational perturbations are needed in order to fit the observational data (Micheli et al. 2012; Farnocchia et al. 2013) as the small size of 2009 BD amplifies the magnitude of nongravitational perturbations, which are inversely proportional to the diameter $D$.

Similarly to Marsden et al. (1973), we modeled nongravitational perturbations as

$$
\mathbf{a}_{N G}=\left(A_{1} \hat{\mathbf{r}}+A_{2} \hat{\mathbf{t}}\right)(1 \mathrm{au} / r)^{2}
$$

where $\hat{\mathbf{r}}$ and $\hat{\mathbf{t}}$ are the radial and transverse directions, respectively, and $r$ is the heliocentric distance. $A_{2}$ is related to the transverse component of the Yarkovsky effect while $A_{1}$ accounts for solar radiation pressure and the radial component of the Yarkovsky effect.

We included $A_{1}$ and $A_{2}$ in the list of parameters estimated from the orbital fit and obtained $A_{1}=(57 \pm 8) \times 10^{-12} \mathrm{au} / \mathrm{d}^{2}$ and $A_{2}=(-113 \pm 8) \times 10^{-14} \mathrm{au} / \mathrm{d}^{2}$. The values of $A_{1}$ and $A_{2}$ along with their uncertainties affect the ephemeris predictions of $2009 \mathrm{BD}$ and provide constraints on its physical properties. In particular, since $A_{2}$ is proportional to $\cos \gamma$, where $\gamma$ is the obliquity (Farnocchia et al. 2013), the negative value of $A_{2}$ implies that $2009 \mathrm{BD}$ is a retrograde rotator.

\section{Spitzer observations}

To obtain additional information on its physical properties, we observed 2009 BD starting on 2013 October 13 for a total of 25 hours with IRAC (Fazio et al. 2004) on the Spitzer Space Telescope. We selected the observation window according to Spitzer observability, the predicted flux density of the asteroid, and we chose the IRAC channel 2 to maximize our chances of detecting 2009 BD (Mommert et al. 2014).

To make sure that 2009 BD was in the field of view, we computed plane-of-sky position along with the corresponding $3 \sigma$ uncertainty for different settings of the dynamical model: 1) gravitational-only solution, 2) accounting for solar radiation pressure, and 3) including both solar radiation pressure and the Yarkovsky effect. Moreover, we tested different statistical treatments of the astrometry by selecting different outlier rejection thresholds 
(Carpino et al. 2003). All these different predictions fell within 20" in right ascension and 2" in declination, well inside the Spitzer field of view, i.e., $312 " \times 312$ ".

Though we did not detect $2009 \mathrm{BD}$ in the Spitzer field of view, we derived a $0.78 \mu \mathrm{Jy}$ $3 \sigma$ upper bound to the flux density. The resulting constraints on the physical properties of 2009 BD are discussed below.

\section{Nongravitational perturbations and thermophysical modeling}

The lack of a clear detection of 2009 BD in our observations precludes a direct determination of its physical properties. To indirectly constrain the physical properties of 2009 BD, we take a probabilistic approach that combines a thermophysical model with a model of the nongravitational effects on the asteroid's orbit.

For solar radiation pressure we adopt the model by Vokrouhlický \& Milani (2000) whereas for the Yarkovsky effect we use the model approach described by Vokrouhlický et al. (2000). The asteroid is assumed to be spherical and the heat transfer is solved analytically using the linearized heat transfer equation. Using the dependence of solar radiation pressure and the Yarkovsky effect on the physical properties of 2009 BD, the model derives bulk density $\rho$ and thermal inertia $\Gamma$ as a function of $\gamma$, and $D$ as a result of the fit to the available astrometric data.

The thermophysical model approximates the surface temperature distribution to determine the thermal-infrared emission from the surface as a function of the physical properties of $2009 \mathrm{BD}$, e.g., rotation state, thermal inertia, and surface roughness. We assume a spherical shape of $2009 \mathrm{BD}$, i.e., the derived diameter is the one of a sphere with the same cross-sectional area as the real shape of 2009 BD. The model numerically solves the heat transfer equation and computes the IRAC channel 2 in-band flux density. The contribution from reflected solar light is added to the calculated flux density as described by Mueller et al. (2011), assuming an infrared/optical reflectance ratio of 1.4.

In both the orbital and the thermophysical models we adopt an absolute magnitude $H=28.43 \pm 0.12$ (Micheli et al. 2012), a photometric slope parameter $G=0.18 \pm 0.13$ (derived as the average from all $G$ measurements of asteroids in the JPL Small-Body Database), and a rotation period $P=2^{(2 \pm 0.5)} \mathrm{hr}$.

\section{Results}

The mutual dependence among physical properties used by the orbital and the thermophysical model require an iterative solution of the problem. We first sampled the obliquity $\gamma$ from $90^{\circ}$ to $180^{\circ}$. We tested the possible diameters using the thermophysical model, based on an upper-limit flux density measurement (0.78 Jy), and obtained that the diameter of $2009 \mathrm{BD}$ is smaller than $8 \mathrm{~m}$.

For each $D<8 \mathrm{~m}$ and $90^{\circ}<\gamma<180^{\circ}$ we find two solutions for $(\Gamma, \rho)$ from the orbital fit to the astrometry. The first solution displays a low $\Gamma$ of about $10 \mathrm{~J} \mathrm{~m}^{-2} \mathrm{~s}^{-0.5} \mathrm{~K}^{-1}$ with a higher bulk density, whereas the second solution stands out with $\Gamma \sim 1000 \mathrm{~J} \mathrm{~m}^{-2} \mathrm{~s}^{-0.5}$ $\mathrm{K}^{-1}$ and a lower bulk density. Based on the orbital fit $\chi^{2}$, we can also further constrain the obliquity (see Table 1) and confidently rule out that 2009 BD is smaller than $2.6 \mathrm{~m}$.

We use our intermediate results to derive diameter distributions for both solutions according to the thermal inertia constraints and the Spitzer upper-limit flux density measurement. We generate a sample of synthetic objects with pairs $(D, \Gamma)$ and we sample the parameters $(H, G, P, \gamma)$. For the surface roughness we randomly pick one of four different roughness models (see Mueller 2007). We model each synthetic sample object and derive its IRAC in-band flux density combined with contributions from reflected 
Table 1. Physical properties of 2009 BD.

\begin{tabular}{|c|c|c|c|}
\hline & \multicolumn{3}{|c|}{ | Low thermal inertia |High thermal inertia } \\
\hline | Diameter [m] & $2.9 \pm 0.3$ & | & $4 \pm 1$ \\
\hline Albedo & $0.85_{-0.10}^{+0.20}$ & | & $0.45_{-0.15}^{+0.35}$ \\
\hline Obliquity $\left[{ }^{\circ}\right]$ & $170_{-15}^{+10}$ & I & $180_{-5}^{+0}$ \\
\hline Density $\left[\mathrm{g} \mathrm{cm}^{-3}\right]$ & $2.9 \pm 0.5$ & | & $1.7_{-0.4}^{+0.7}$ \\
\hline Mass [t] & $36_{-8}^{+10}$ & | & $55_{-25}^{+30}$ \\
\hline Thermal inertia $\left[\mathrm{J} \mathrm{m}^{-2} \mathrm{~s}^{-0.5} \mathrm{~K}^{-1}\right]$ & $30_{-10}^{+20}$ & I & $2000 \pm 1000$ \\
\hline
\end{tabular}

solar light, which we then compare with the upper-limit flux density as derived from our observations. The final solutions for the diameter are shown in Table 1.

Based on the solution-specific diameter ranges, we finally constrain the other physical properties of 2009 BD using the nongravitational perturbation model and the orbital fit to the astrometry. Table 1 shows the derived physical properties of 2009 BD for both the low and high thermal inertia solutions. The first solution shows 2009 BD as a massive rock body covered with regolith-like material, causing it to exhibit a low thermal inertia. The second solution suggests 2009 BD to be a rubble-pile asteroid that consists of individual bare rock slabs. We are unable to rule out either solution at this stage.

\section{Acknowledgements}

The authors thank Tom Soifer, Director of the Spitzer Space Telescope, for the time allocation to observe 2009 BD. The work of D. Farnocchia and S. R. Chesley was conducted at the Jet Propulsion Laboratory, California Institute of Technology under a contract with NASA. D. Vokrouhlický was partially supported by the Grant Agency of the Czech Republic (grant P209-13-01308S). J. L. Hora and H. A. Smith acknowledge partial support from Jet Propulsion Laboratory RSA No. 1367413. This work is based on observations made with the Spitzer Space Telescope, which is operated by the Jet Propulsion Laboratory, California Institute of Technology under a contract with NASA. Copyright 2014 California Institute of Technology.

\section{References}

Buzzi, L., Hormuth, F., Bittesini, L., et al. 2009, MPEC, 2009-B14

Carpino, M., Milani, A., \& Chesley, S. R. 2003, Icarus, 166, 248

Farnocchia, D., Chesley, S. R., Vokrouhlický, D., et al. 2013, Icarus, 224, 1

Fazio, G. G., Hora, J. L., Allen, L. E., et al. 2004, ApJS, 154, 10

Marsden, B. G., Sekanina, Z., \& Yeomans, D. K. 1973, AJ, 78, 211

Mazanek, D. D., Brophy, J. R., \& Merrill, R. G. 2013, Planetary Defense Conference, IAAPDC13-04-14

Micheli, M., Tholen, D. J., \& Elliott, G. T. 2012, New Astron., 17, 446

Mommert, M., Hora, J. L., Farnocchia, D., et al. 2014, ApJ, 786, 148

Mueller, M. 2007, arXiv, 1208.3993

Mueller, M., Delbo', M., Hora, J. L., et al. 2011, AJ, 141, 109

Tholen, D. J., Micheli, M., Bauer, J., \& Mainzer, A. 2013, AAS/Division for Planetary Sciences Meeting Abstracts, 45, \#101.08

Vokrouhlický, D. \& Milani, A. 2000, A\&A, 362, 746

Vokrouhlický, D., Milani, A., \& Chesley, S. R. 2000, Icarus, 148, 118

Werner, M. W., Roellig, T. L., Low, F. J., et al. 2004, ApJS, 154, 1 